\title{
Detection of Fingerprint using He-Ne and Diode Lasers on Aluminium and Glass Surfaces
}

\author{
Ali Abdelrhman Saeed Marouf ${ }^{1,}$, , Malka Aldar Daood Abdalrheim Daood ${ }^{2}$ \\ ${ }^{1}$ Institute of Laser, Sudan University of Science and Technology, Khartoum, Sudan \\ ${ }^{2}$ College of Science, Sudan University of Science and Technology, Khartoum, Sudan
}

Email address:

marou.44@gmail.com (A. A. S. Marouf), alisaeed@sustech.edu (A. A. S. Marouf), nanoshdaood45@gmail.com (M. A. D. A. Daood)

${ }^{*}$ Corresponding author

\section{To cite this article:}

Ali Abdelrhman Saeed Marouf, Malka Aldar Daood Abdalrheim Daood. Detection of Fingerprint using He-Ne and Diode Lasers on Aluminium and Glass Surfaces. Radiation Science and Technology. Vol. 5, No. 4, 2019, pp. 37-40. doi: 10.11648/j.rst.20190504.11

Received: October 1, 2019; Accepted: October 22, 2019; Published: October 31, 2019

\begin{abstract}
There are different latent fingerprint recovering techniques, physical and chemical techniques are available for the development of latent fingerprints. Fingerprints detection requires high sensitivity, which is provided by luminescence and appropriate physical and chemical treatments. Photoluminescence detection of latent fingerprints has over the last quartercentury brought about a new level of fingerprint detection sensitivity. Fingerprints have many uses, a chemical solution usually used to detect fingerprints but it takes time. In this paper, we were operating with low-power lasers, He-Ne laser with output power $1 \mathrm{mmW}$ and wavelength $632.8 \mathrm{~nm}$ and diode laser with output power $5 \mathrm{mmW}$ and wavelength $532 \mathrm{~nm}$ to detect fingerprints on an aluminium and glass surfaces. Fingerprints were exposed by the laser beam through a convex lens with focal length $5 \mathrm{~mm}$ then a digital camera was used to record luminescence from fingerprint. The revealed results show clear images for the fingerprints using two types of lasers He-Ne and diode and appearing its characteristics on different surfaces obviously. Using laser sources to detect fingerprints is a simple method using low-level lasers without chemicals, in a short period of time, with low cost and gives good results.
\end{abstract}

Keywords: Forensic Science, Laser-matter Interaction, Laser Luminescence, Latent Fingerprint Detection, Photoluminescence, Optical Sensor

\section{Introduction}

Significant advances in laser technology and the development of novel light detectors have dramatically improved spectroscopic methods for molecular characterization over the last decade. Lasers with its different spectral region can insert in many applications several among many applications, the use of lasers in optical disk drives, laser printers and engines, barcode scanners and fibre optic in a free-space optical communication, laser surgery and skin treatment, cutting and welding materials; military and law enforcement agencies to identify goals and measure their speed and laser lighting displays in the field of entertainment DNA sequencing tools used to detect fingerprints. [1-4].

When a finger touches a surface, on the order of $0.1 \mathrm{mg}$ of residue is left behind, comprising a latent fingerprint. Typically, $98-99 \%$ of this is water, which soon evaporates to leave behind on the order of a microgram of material, which is made up of, in roughly equal quantities, inorganic salts and a complex mixture of organic constituents, including fatty acids, triglycerides, amino acids, vitamins, squalene, urea, etc. The visualization of latent fingerprints mostly involves targeting a class of these organic components.

Different powders and Small particle reagent (SPR) have been used by various workers for the development of latent fingerprints on different surfaces. [5-11].

Several researchers investigated whether certain fingerprint methods can recover latent fingerprints on glass and metal surfaces submerged in water. $[8,10,11]$

The light sources used to generate narrow bandwidths come in several different varieties, including UV lamps, filtered lamps, and lasers. Each of these light sources has advantages and disadvantages, depending on the intended purpose and one's budget. Recently, "alternate" or "forensic" light sources (filtered lamps) have become heavily relied on 
in laboratories and at crime scenes because of improvements in power output, versatility, portability, and affordability when compared to lasers.

In 2006 M. T. TASCHUK et al conducted a preliminary investigation of the capabilities of femtosecond LIBS for detection and mapping of latent fingerprints, and demonstrated its structure using 400nm, $84 \pm 7 \mu \mathrm{J}$ pulses.[12]. Also in 2009, Alaoui used Argon laser with the blue lines 476.5 and $488 \mathrm{~nm}$ to excited the prints to be detected and observed the sample's fluorescence is under orange filters. He also compared between photoluminescence fingerprints and trace explosives detection. [13].

In this paper, we have studied visible fluorescence spectra of fingerprints excited with $532 \mathrm{~nm}$ and $632 \mathrm{~nm}$ illumination in detail with our new imaging system.

\section{Materials and Methods}

The first laser used to detect the fingerprint was HeliumNeon laser (He-Ne) with wavelength $632.8 \mathrm{~nm}$ and output power $1 \mathrm{~mW}$, the second one was green diode laser pointer with wavelength $532 \mathrm{~nm}$ and output power $5 \mathrm{~mW}$. Convexes lens with focal lens $5 \mathrm{~mm}$ was used to defocuses laser and carried out the photoluminescence from the surfaces. A note3 phone camera with megapixel $13 \mathrm{mp}$ and $2160 \mathrm{p}$ was used to photo the fingerprint. Two different surfaces were used to detect the fingerprint, the first sample was an aluminium knife with a soft surface, the second surfaces were microscopic slides with clear glass and thickness of $1.0 \mathrm{~mm}-$ $1.2 \mathrm{~mm}$ and $25 \times 75 \mathrm{~mm}$ dimensions.

Fingerprints deposited on the two different dry surfaces (aluminium and glass); two impressions were taken from person on the two surfaces; each impression consisted of pressing the pads of the four fingers onto the surface. Surfaces were excited by laser beams with different powers and wavelengths through convex lens, with focal length $5 \mathrm{~mm}$. Diode laser (with wavelength $532 \mathrm{~nm}$ and output power $5 \mathrm{~mW}$ ) and $\mathrm{He}-\mathrm{Ne}$ laser (with wavelength $632.8 \mathrm{~nm}$ and output power $1 \mathrm{~mW}$ ) were used for this purpose. The search for luminescence from the samples was undertaken without dyes, then a photo of these fingerprints were recorded by digital mobile phone camera without using a Polaroid, with appropriate angle, see figure 1 .

Lasers can generate enough intensity that even incidental or reflected light may damage the unprotected eye; so goggles were weird.

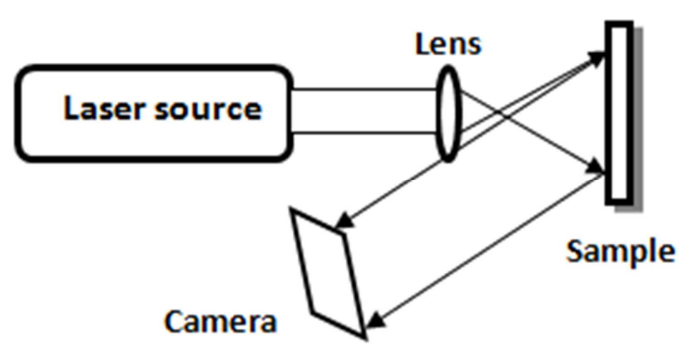

Figure 1. Configuration used to record photographs of luminescent fingerprints; schematic diagram.

\section{Results}

\subsection{Detection of Fingerprint on Aluminium Surface}

Figure 2 shows a photo of fingerprint that has been appearing by red laser excitation with low output power $1 \mathrm{~mW}$ on an aluminium knife surface.

When $\mathrm{He}-\mathrm{Ne}$ laser with output power of $1 \mathrm{~mW}$ exposed on aluminium knife surface a fingerprint with six different patterns appears, with the following characteristics:
1. Island.
2. Core.
3. Bridge.
4. Bifurcation.
5. Ending ridge.
6. Crossover.

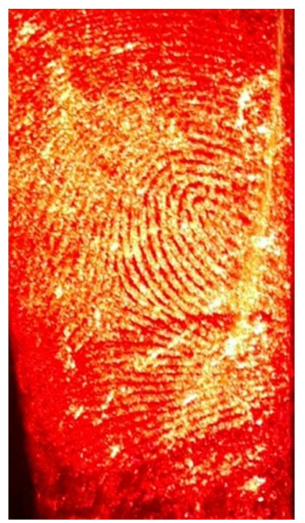

Figure 2. Fingerprint mark recovery from metal (aluminium) surface by red laser.

Figure 3 shows a photo of another different fingerprint that has been appearing by green laser excitation with low output power $5 \mathrm{~mW}$ on an aluminium knife surface.

Diode laser pointer with an output power of $5 \mathrm{~mW}$ used to expose on aluminium knife surface the showing fingerprint with six different following patterns:
1. Island.
2. Core.
3. Bridge.
4. Bifurcation.
5. Ending ridge.
6. Crossover.

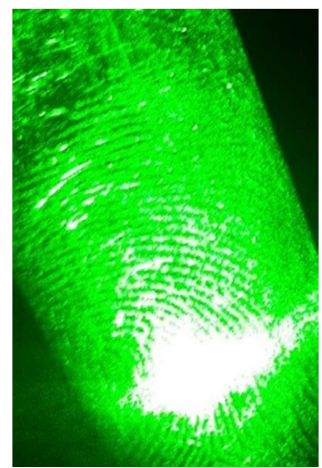

Figure 3. Fingerprint mark recovery from metal (aluminium) surface by green laser. 


\subsection{Detection of Fingerprint on Glass Surface}

Figure 4 shows a photo of fingerprint that has been appearing by laser exposure with power $1 \mathrm{~mW}$ on a microscopic glass slide surface.

When He-Ne laser with output power of $1 \mathrm{~mW}$ exposed on microscopic glass slide surface a fingerprint with seven different patterns appears, with the following characteristics:
1. Island.
2. Core.
3. Bridge.
4. Bifurcation.
5. Ending ridge.
6. Crossover.
7. Delta.

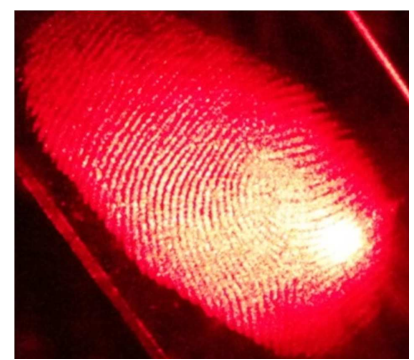

Figure 4. Fingerprint mark recovery from glass surface by red laser.

Figure 5 shows a photo of the same fingerprint that has been appearing by laser exposure with power $5 \mathrm{~mW}$ on a microscopic glass slide surface.

Diode laser pointer with an output power of $5 \mathrm{~mW}$ used to expose on microscopic glass surface the showing fingerprint with seven different following patterns:
1. Island.
2. Core.
3. Bridge.
4. Bifurcation.
5. Ending ridge.
6. Crossover.
7. Delta.

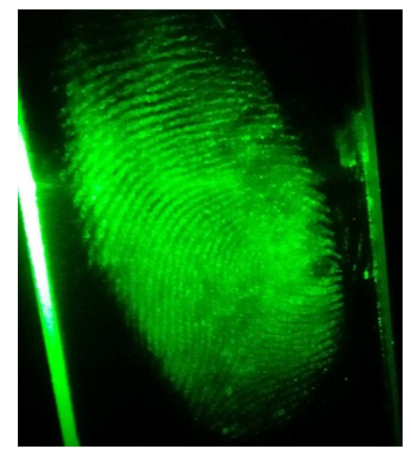

Figure 5. Fingerprint mark recovery from glass surface by green laser.

\section{Discussion}

Viewing fingerprints under a laser could induce fluorescence prints. The fingerprint fluorescent images on a metallic surface using red and green laser beams presented in figures 2 and 3, and the fingerprint images on a glass surface using red and green laser beams presented in figures 4 and 5 . These images represent approximately $85 \%$ of a total thumbprint area of approximately $6 \mathrm{~cm}^{2}$; they show a lot of patterns. There is no possibility of unacceptable damage to the fingerprint when imaging it by these visible lasers. Laserinduced fluorescence is generally considered a nondestructive technique. In comparison by using Laser-Induced Breakdown Spectroscopy, small damage will happen to the print to the point that the LIBS measurement process. [12]

Good quality fluorescent images of a latent fingerprint could be successfully imaged using this technique. This simple system has been successful in ablating finger marks on glass and aluminium; it can be applied to detect the fingerprints from the surfaces which it is notoriously difficult to lift fingerprints with results that are better or comparable to traditional methods.

\section{Conclusions}

In conclusion, fingerprints were shown on the surfaces of aluminium and glass using a $\mathrm{He}-\mathrm{Ne}$ and diode lasers with output power of $1 \mathrm{~mW}$ and $5 \mathrm{~mW}$ respectively, the results revealed a reliable fingerprint signals on different surface materials in a short time, with no damage to the latent fingerprint and less cost than chemical demonstration methods. Further study is expected to study and compare two different visible laser light sources based on portability, cost, sensitivity, and power output.

\section{Acknowledgements}

The authors would like to thank the Institute of Laser at Sudan University of Science and Technology (SUST) and all members there for performing this work.

\section{References}

[1] Saitoh N, Akiba N. Ultraviolet fluorescence spectra of fingerprints. The Scientific World Journal. 2005; 5: 355-66.

[2] Dalrymple BE, Duff JM, Menzel ER. Inherent fingerprint luminescence-detection by laser. Journal of Forensic Science. 1977 Jan 1; 22 (1): 106-15.

[3] Menzel ER, Savoy SM, Ulvick SJ, Cheng KH, Murdock RH, Sudduth MR. Photoluminescent semiconductor nanocrystals for fingerprint detection. Journal of Forensic Science. 2000 May 1; 45 (3): 545-51.

[4] Gaensslen RE, Ramotowski R, Lee HC. Advances in fingerprint technology. CRC press; 2001 Jun 15.

[5] Menzel ER. Fingerprint Detection with Photoluminescent. Advances in Fingerprint Technology. 2001 Jun 15: 211.

[6] Rohatgi R, Kapoor AK. Development of latent fingerprints on wet non-porous surfaces with SPR based on basic fuchsin dye. Egyptian Journal of Forensic Sciences. 2016 Jun 1; 6 (2): 17984. 
[7] Dhall JK, Sodhi GS, Kapoor AK. A novel method for the development of latent fingerprints recovered from arson simulation. Egyptian Journal of Forensic Sciences. 2013 Dec 1; 3 (4): 99-103.

[8] Trapecar M. Fingermarks on glass and metal surfaces recovered from stagnant water. Egyptian Journal of Forensic Sciences. 2012 Jun 1; 2 (2): 48-53.

[9] Bumbrah GS. Small particle reagent (SPR) method for detection of latent fingermarks: A review. Egyptian Journal of Forensic Sciences. 2016 Dec 1; 6 (4): 328-32.

[10] Badiye A, Kapoor N. Efficacy of Robin ${ }^{\circledR}$ powder blue for latent fingerprint development on various surfaces. Egyptian Journal of Forensic Sciences. 2015 Dec 1; 5 (4): 166-73.
[11] Jasuja OP, Kumar P, Singh G. Development of latent fingermarks on surfaces submerged in water: Optimization studies for phase transfer catalyst (PTC) based reagents. Science \& Justice. 2015 Sep 1; 55 (5): 335-42.

[12] Taschuk, M. T., Tsui, Y. Y., \& Fedosejevs, R. (2006). Detection and mapping of latent fingerprints by laser-induced breakdown spectroscopy. Applied spectroscopy, 60 (11), 13221327.

[13] Alaoui, I. M. (2009). Applications of luminescence to fingerprints and trace explosives detection. In Unexploded Ordnance Detection and Mitigation (pp. 189-196). Springer, Dordrecht. 